\title{
The art of virtual mentoring in the twenty-first century for STEM majors and beyond
}

\author{
Social quarantine has severely impacted the training of new scientists, but the use of virtual platforms can \\ supplement mentorship, making career development seamless.
}

\begin{abstract}
ocial distancing restrictions have severely impacted the laboratory environment by limiting in-person activities. As a result of reduced in-person interactions between trainees, mentors and staff, the dynamic of traditional mentormentee relationships has also become remodeled (Fig. 1), leading many to rely on mentorship undertaken through virtual platforms. For the majority of trainees and mentors, mentorship that relies primarily on virtual platforms is an unfamiliar concept. If this unfamiliar mentoring relationship is not navigated properly, there can be significant consequences for the development of the trainee and productivity of the mentor. Here, we provide examples of how to engage in a virtual mentoring relationship to maintain motivation, team cohesion and research productivity, with examples from academia and the biotech industry. We argue that virtual mentoring allows mentors to intentionally support their trainees through scheduled interactions using digital platforms to facilitate growth and career development during these uncertain times.
\end{abstract}

\section{What is virtual mentoring?}

Virtual mentoring enables the exchange of information in the digital realm through the use of one or more digital platforms (Table 1). To help trainees achieve success, virtual mentoring supports skillset development, knowledge acquisition, confidence building and cultural competence while using a platform to generate and nurture relationships between mentors and mentees. During the COVID-19 pandemic, virtual mentoring endeavors can forge productive and substantial interconnections between mentors and trainees that can further develop scientific and professional skill sets. Figure 2 shows such a virtual mentoring session on the Zoom platform.

Virtual mentoring provides a unique set of pros and some cons that can respectively support or hinder mentorship activities (Table 2). Have you ever abstained from asking a question in a lab meeting or a

In-person

Virtual

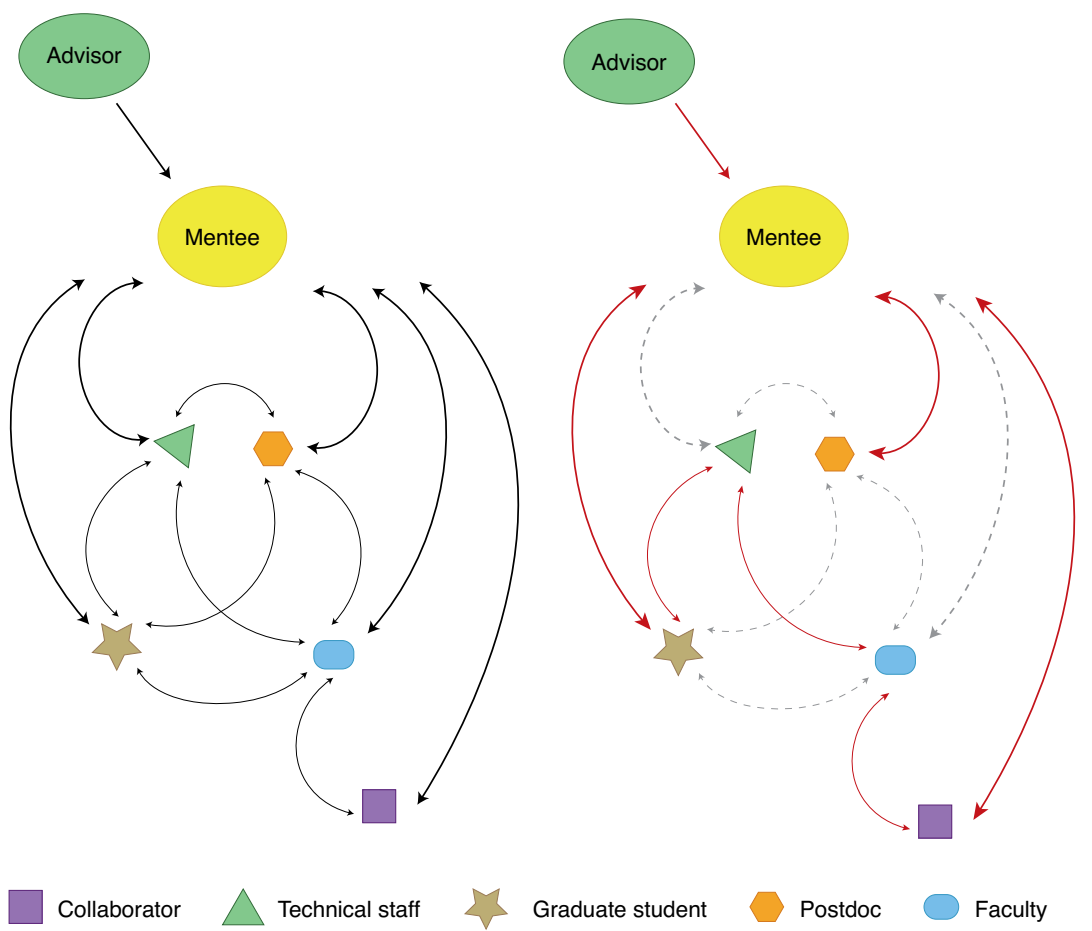

Fig. 1 | In-person vs. virtual mentoring networks. The dynamic mentoring interactome generated by in-person and virtual mentoring experiences. While virtual mentoring can limit the number and frequency of interactions, this platform does still support a productive training environment for trainees while providing resources for mentors to extend their mentorship. Black arrows represent in-person interactions, red arrows indicate virtual interactions and gray dashed arrows represent missed interactions.

seminar presentation for fear of sounding uninformed? Is there a topic that you wanted to dive into with a mentor but were afraid to take up their time? Virtual mentoring creates an opportunity for mentors to support trainees exploring new research directions built from the digital realm. For example, screen sharing allows mentors a platform to educate mentees on techniques in computational biology, statistical analysis and bioinformatics by directly training scientists in mentoring sessions. Virtual mentoring sessions can also provide a less judgmental learning environment than in-person mentoring meetings by reducing all involved parties to a voice and a screen.

Mentoring, whether virtually or in person, has clear benefits for career development. Virtual mentoring allows people to more easily accommodate the busy schedules of multiple mentors and eliminates the hindrance of space availability associated with in-person meetings. Having multiple mentoring relationships, or a mentoring network, exposes trainees to a diversity of ideas and advice from people with varying backgrounds and scientific expertise and is vital for career development and problem-solving ${ }^{1-4}$. The effects of 
Table 1 | Virtual mentoring platforms, with application type and features

\begin{tabular}{|c|c|c|}
\hline Platform & Type & Virtual mentoring features \\
\hline Zoom & $\begin{array}{l}\text { Audio and video } \\
\text { call application }\end{array}$ & $\begin{array}{l}\text { Waiting room feature, calendar integration, multi-screen share, } \\
\text { free basic interface, chat feature }\end{array}$ \\
\hline Skype & $\begin{array}{l}\text { Audio and video } \\
\text { call application }\end{array}$ & $\begin{array}{l}\text { Messaging, screen sharing, recording capabilities; can call } \\
\text { phones; free basic interface }\end{array}$ \\
\hline Trello & $\begin{array}{l}\text { Project management } \\
\text { tool }\end{array}$ & $\begin{array}{l}\text { Free for basic service; simple sharing of tasks including calendar } \\
\text { and deadlines }\end{array}$ \\
\hline Slack & $\begin{array}{l}\text { Collaboration } \\
\text { software }\end{array}$ & $\begin{array}{l}\text { Organized chat conversations by topics; team members can } \\
\text { share files; face-to-face or chat option }\end{array}$ \\
\hline Blue Jeans & $\begin{array}{l}\text { Video conferencing } \\
\text { application }\end{array}$ & $\begin{array}{l}\text { Audio or video conferencing option, scheduling and productivity } \\
\text { tools }\end{array}$ \\
\hline Twitter & $\begin{array}{l}\text { Social media } \\
\text { platform }\end{array}$ & $\begin{array}{l}\text { Tweet threading for themed information sharing; mentorship } \\
\text { extended to broad group of followers; direct messaging option }\end{array}$ \\
\hline Facebook & $\begin{array}{l}\text { Social media } \\
\text { platform }\end{array}$ & $\begin{array}{l}\text { Information sharing, direct messaging option, option to join } \\
\text { mentoring groups }\end{array}$ \\
\hline WeChat & Messaging app & Overseas favorite \\
\hline WhatsApp & Messaging app & End-to-end encryption \\
\hline QQ & Messaging app & $\begin{array}{l}\text { Most popular video/chatting app in China; connects } \\
\text { internationally and has both phone and computer interfaces }\end{array}$ \\
\hline LINE & Messaging app & Video and text messaging, games, voice calls \\
\hline KAIKO talk & Messaging app & $\begin{array}{l}\text { Video and text messaging, file sending, remote logout for PC } \\
\text { version, voice calls, calendar }\end{array}$ \\
\hline
\end{tabular}

multiple mentoring relationships on career development has been demonstrated in the workplace and results in more significant commitment and higher job satisfaction ${ }^{5}$. Researchers have also demonstrated that women who have access to mentoring and more training opportunities attain reflective power that improves performance faster because of access to better resources and constructive feedback ${ }^{6,7}$.

\section{Facilitating virtual mentoring sessions}

How do we initiate and facilitate a virtual mentoring session? We describe a protocol and considerations for undertaking a virtual mentoring meeting (Fig. 3). One advantage of virtual meetings is that, in general, they tend to run more efficiently than in-person meetings. There is something about the idea that the other party can disconnect and disappear at any moment that stimulates productivity.

Setting up the virtual mentoring space. As eye contact is an important communication mechanism that is naturally available during in-person meetings, we suggest using a web camera to support this interaction. To mimic a traditional meeting space, we recommend using the web camera attached to the screen where the meeting takes place, rather than a second monitor in which you may not be making eye contact regularly. By creating a more natural environment, we expect to generate more engagement and ultimately, improved communication.

The meeting environment is further complicated by the user background, which during virtual sessions may be in their home or another remote working location. Being mindful of how your background may influence the meeting atmosphere is essential to maintaining professionalism and reducing any discomfort that other participants may feel. One alternative that can help is to generate a professional background using the Zoom and BlueJeans platforms, which allows users to employ template backgrounds to eliminate unnecessary attention that may take away from the productivity of a virtual mentoring session.

Agenda. Due to the strict scheduling constraints of virtual mentoring sessions, we strongly suggest generating an agenda to facilitate the mentoring session. For novice mentees such as undergraduate students, it may be necessary for the mentor to ask the mentee to generate an agenda before the meeting. For more experienced mentees such as postdoctoral fellows, it would be expected that the trainee creates an agenda before the virtual meeting. However, in some instances, the mentor may generate the agenda to ensure that their needs are also being met. Once the purpose of the meeting has been communicated digitally and the meeting has been scheduled, the session can begin. It is customary to begin meetings with small talk to settle in and build rapport. For example, "How is the weather? Have you done anything fun today?" Breaking the ice may allow the mentor and mentee to feel more comfortable sharing information throughout the meeting.

You are now ready to move on to business. Discussions during the virtual mentoring sessions can range across any number of topics that would normally be covered during an in-person session or informal meeting. For example, discussions of the status of current or future projects, manuscripts in progress, upcoming presentations or job applications are likely topics for a virtual mentoring session. Many labs also use virtual workspaces to conduct journal clubs to facilitate the development of presentation and discussion skills on the part of trainees. Holding journal club discussions within the digital realm lets researchers access at their fingertips a plethora of scientific resources that can enable a deeper discussion of the specifics of a publication. It also allows novice trainees to look up unfamiliar concepts during the discussion, supporting their scientific development in real time.

Following discussions about projects and papers, mentors and mentees may identify roadblocks to success. In the case of logistical barriers, a trainee may need help contacting a collaborator or scheduling time on a new piece of equipment. However, the most significant and overlooked roadblocks are frequently internal - for example, the mental and emotional well-being of trainees. It is hard enough to address this type of roadblock in person, let alone from afar. So, how do mentors address internal challenges in the age of social isolation?

Tell stories of failure before success. As scientific mentors, we can use personal stories to teach trainees that failures will occur along the road to success. In the words of Thomas Edison, "Many of life's failures are people who did not realize how close they were to success when they gave up." One of the most common causes of distress in young scientists is a misalignment of expectations with reality. Experiments gone haywire, grant rejections and interactions with reviewer no. 3 are common experiences that every scientist has navigated through at some point along their career and learned to overcome. Yet, despite these shared experiences, the stories that we hear from the greater scientific community are skewed almost exclusively in favor of success. Accepted publications, funded grants and career awards are celebrated, while negative 


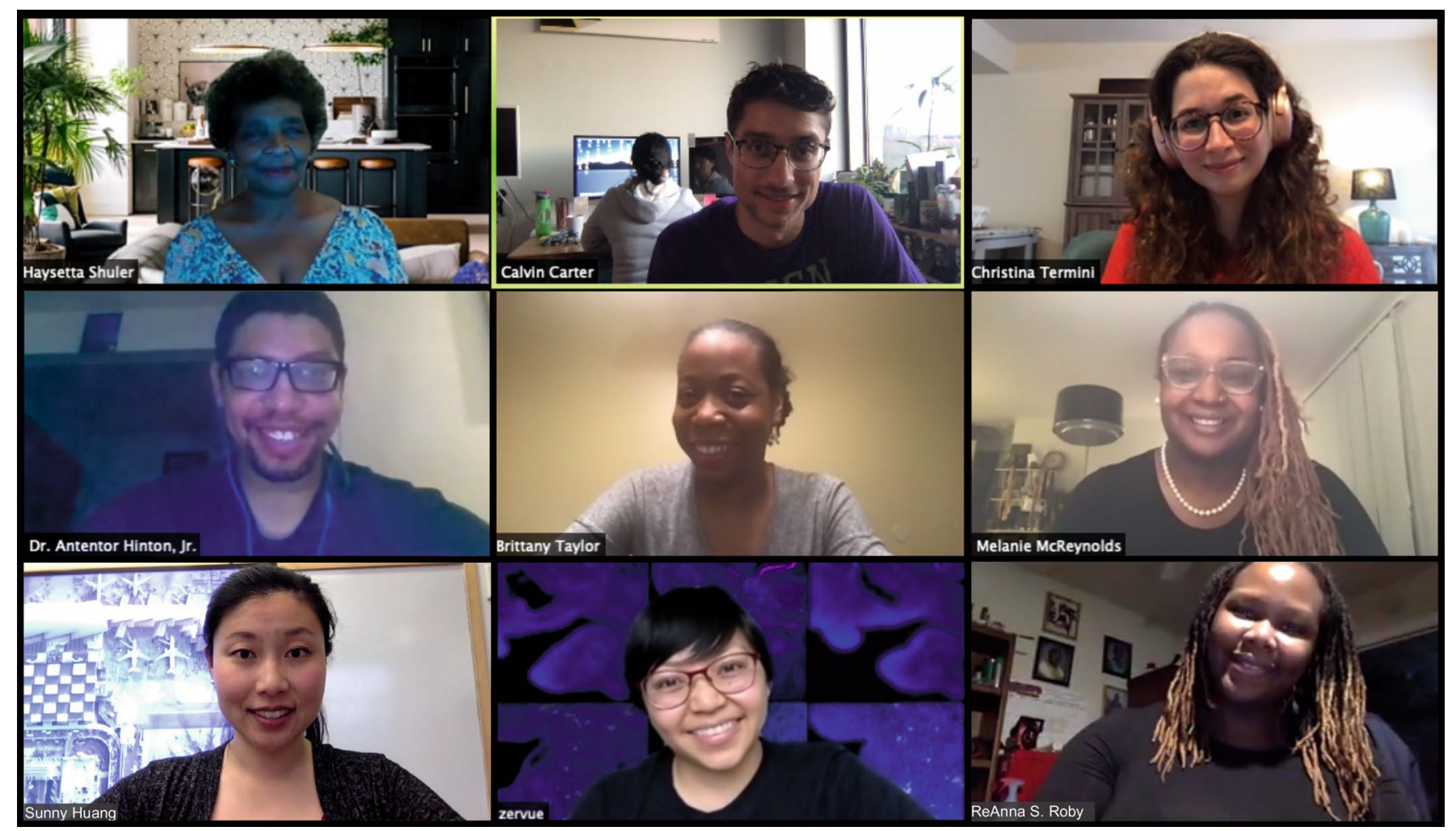

Fig. 2 | Mentoring session in action. Mentees from the University of lowa, University of Pennsylvania, Princeton University, Vanderbilt University, University of California, San Francisco and University of California, Los Angeles meet virtually using the Zoom digital platform. Under the mentorship of Haysetta Shuler of Winston-Salem State University, the mentees receive critical feedback regarding their writing and develop an action plan to meet their goals.

data are rarely published and rejected grant proposals are relegated to our computers. This imbalance is unrealistic and can be demotivating to any scientist, especially for trainees who may not yet have the context or the skill set to understand and deal with the daily realities of researching. It is important that these same struggles and failures be communicated from mentors to mentees in virtual mentoring sessions. Not every mentee needs to hear a sublime story about how things worked, but what failed and how you actually were able to work through a cumbersome challenge can inspire mentee perseverance. The ability to have grit behind a computer or in a professional workplace is what helps trainees develop into the next generation of scientific leaders.

Notably, a lesson in managing expectations can be learned from the startup world. Silicon Valley accelerators have learned that one of the best strategies to teach young startups the skills to persevere through failures is to normalize failure through stories. In other words, they expose inexperienced founders to the necessity of failure. Every successful founder has experienced hundreds if not thousands of challenges, mistakes and failures that have put their businesses and careers on the brink of death. Accelerators invite successful founders to share intimate stories of their failures with young and inexperienced founders. In private settings, successful founders reveal the embarrassing details of their often outrageous journeys toward success. Founders describe seemingly insurmountable situations and feelings of utter helplessness almost as the norm yet, through sheer will and determination, they persevere in the face of extreme uncertainty. Especially during this difficult time for early career scientists, we encourage mentors to share stories of failure help to reassure mentees that setbacks are part of the journey toward success.

Build your team. We also challenge mentors to form breakout groups and invite multiple mentors to develop a mentoring team. A multi-mentor network allows a mentee to access the most promising traits and experiences from each mentor and allows mentors to learn from others.

Maintaining motivation and team cohesion from afar is another crucial but challenging task. Extra effort must be made to support group unity during times of isolation. To demonstrate to our teams that we are here for one another, we encourage participation in non-academic virtual activities, also known as 'play-centric meetings'. Play-centric meetings are team-building exercises that can be conducted virtually to develop trainees in areas that may complement their academic work. Think of these exercises as 'cross-training' for building teams. These can be conducted toward the end of work-centric meetings or can be conducted in separate meetings.

At the University of Iowa, the Medical Scientist Training Program (MSTP) program exemplifies this approach. During social isolation, MSTP students play games together such as Zoom trivia. Another favorite game is Agar.io, a game in which you play as bacteria looking to take over the Petri dish. Teamwork is essential as you compete with players from around the world. These team-building exercises keep cohesion secure and allow for play, which, with hard work, is one half of the equation to success.

Another option for a low-stress way to communicate with teams while in social quarantine is to host a Zoom coffee, tea or spicy beverage hour. Pick a topic and converse. At the University of Pennsylvania, postdoctoral trainees in the Department 


\section{Table 2 | Pros and cons of virtual mentoring}

Pros Cons

Flexible and time-efficient

Requires stable internet connection and a working electronic device

Less judgmental form of communication

Meetings can be recorded (obtain permission)

Lack confidentiality (see Zoom bombing)

Virtual meetings may not capture the ambience of in-person meetings

Chat feature for general and direct messages

Participants may experience discomfort sharing their surrounding environment

Allows for useful multitasking and immediate information sharing

Partaking in virtual meetings may intimidate participants, especially those who are shy

Meeting participants are not limited by physical distance

Accurate automated written transcription

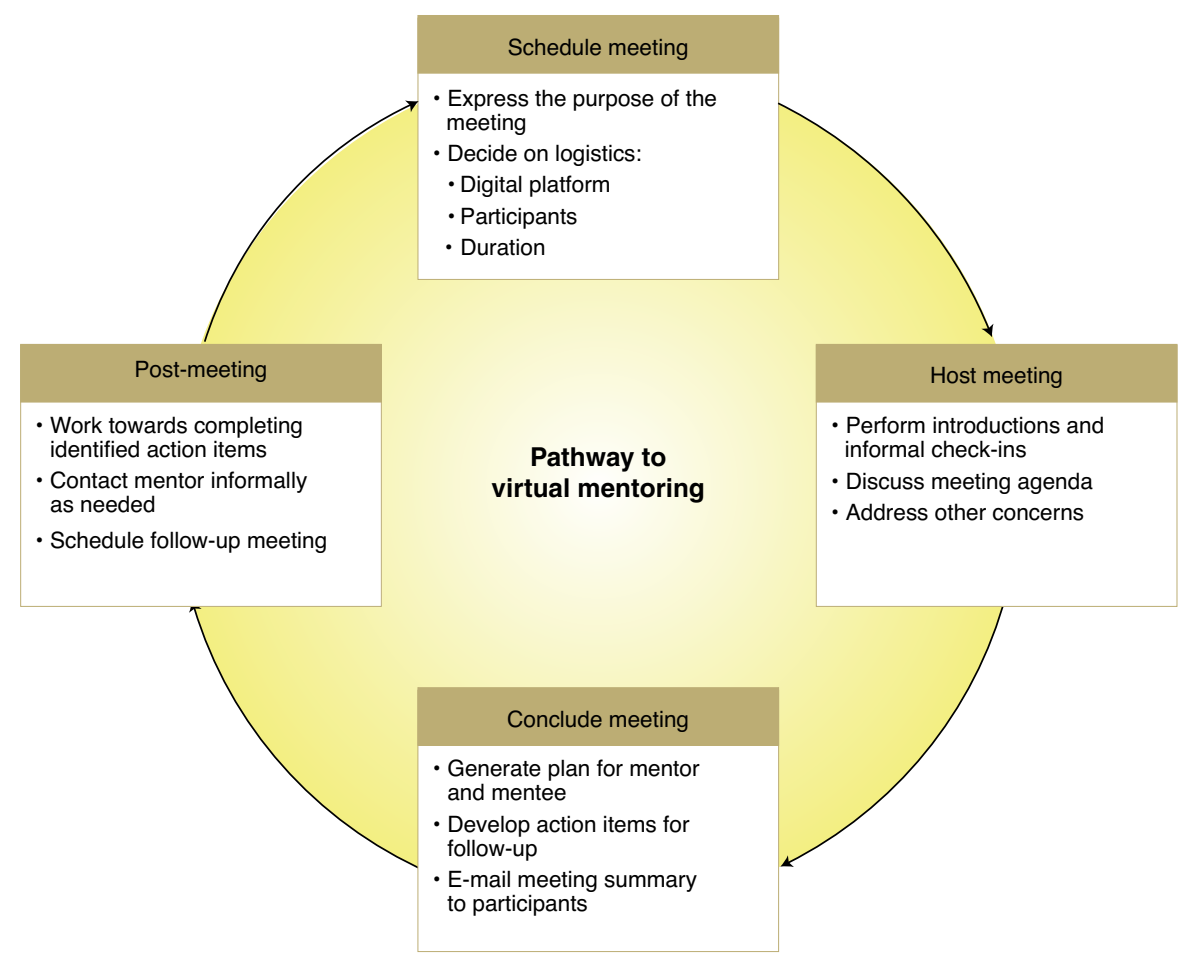

Fig. 3 | Pathway to virtual mentoring. Key steps outlining the fundamentals and protocol for virtual mentoring meetings. Because of the intent and commitment required for a virtual meeting, they are often more efficient than in-person meetings. By providing trainees with the responsibility of generating an agenda at an early stage in their careers, they will gain skills in organization, self-advocacy and accountability. Mentors can use these meetings to ensure continued scientific progress by their mentees while providing necessary feedback and assurance to their trainees. By working together to generate a meeting summary and specific goals for the next meeting, the mentor and mentee clearly articulate their expectations, further supporting a successful virtual mentorship.

of Orthopedic Surgery put together weekly social and professional development activities. The social activities include virtual yoga sessions, virtual lunches and social hours with various principal investigators in the department. Additionally, participants engage in activities and competition to raise money for a local organization providing support to individuals and families affected by the pandemic. When choosing activities, be creative and get a feel of what excites your trainees. Tie the activities into the community so that trainees feel that not only are they engaging with their colleagues, but they are also impacting the larger community.

Ask about their well-being. Being a scientist at any training level can be mentally and emotionally challenging under normal circumstances. Being socially isolated can make it even more difficult to manage these aspects of well-being. We challenge mentors to ask their trainees about their well-being during virtual mentoring sessions and offer to listen. It is important to find an appropriate time to discuss these sensitive but important subjects. For example, asking many trainees about their emotional well-being during a large lab meeting may not be well received. A trainee will feel much more comfortable opening up about these struggles in a smaller group setting or a one-on-one meeting. As mentors, be prepared to hold emotional space for mentees if they do open up about their struggles. Prepare a few questions that show curiosity about their emotions, concerns and challenges. As a mentor, we don't have to solve a mentee's problems - sometimes all they need is someone to actively listen.

In the event that a mentor does not feel comfortable supporting their mentees in this way, there are many options to get a mentee the help they need. For example, the University of Iowa MSTP holds student-run sessions where trainees can freely talk about emotional struggles and concerns that are then brought to the directors (mentors) in an anonymous format for guidance and assistance. Additionally, the mentor can further encourage the mentee to consider seeking more specialized mental health sessions to support their overall well-being, particularly during these difficult times.

End the meeting. Finally, it is important to leave off on a positive note to set an enthusiastic tone for the team. Tell a story or make a joke. Be vulnerable and help maintain a safe space for open and honest discussions.

\section{How long and how often?}

The frequency of virtual mentoring meetings will vary according to the career stage, level of independence and needs of the mentee. For example, a mentor may meet more frequently with early-stage graduate students to ensure they are receiving the feedback and input required to acclimate them to their research projects, which may still be unfamiliar territory. By contrast, a mentor may meet less frequently with senior postdoctoral fellows who have already demonstrated independence and direction 
in their research training. Another factor that influences the frequency of meetings is the needs of the mentor. For example, if a mentee is working with a mentor on a publication, it may be necessary to hold more frequent virtual meetings to check in as new data accumulates, as this may affect the direction of the publication. More frequent meetings may also allow the mentor and mentee to hold each other accountable for their respective contributions, which may help ensure that project deadlines are met. Beyond the realm of productivity, holding frequent meetings allows mentors to reassure their trainees and demonstrate that mentors care about the well-being and success of their trainees.

\section{Challenges in virtual mentor-mentee relationships}

Even in the most successful mentoring relationships, challenges exist. This is also true of virtual mentoring, with the added complexity of relying solely on electronic interactions. Access of resources such as electronics, learning space, bandwidth capacity and even internet are certainly disproportionate across various groups. Therefore, we must consider and address issues of equity that are alleviated and/or exacerbated through virtual mentoring particularly for mentees who have different obligations as a result of returning home during this time.

Building rapport is essential to supporting any mentoring relationship. One way to build rapport is to discuss common interests to establish trust, which can be used to support virtual mentorship. In a virtual environment, it may be difficult to build rapport as meetings may be strictly focused on business. We suggest that mentors and mentees engage in casual conversation in addition to the items on their agenda. To further support trust, we suggest establishing boundaries before holding a virtual mentoring session. For example, it may be necessary to ask whether it is okay to record the session or whether any information discussed should remain confidential. These seemingly small adjustments to protect the confidentiality and privacy of a mentee or mentor can support the mental and emotional health of the trainee to enable a proactive and productive relationship.

Most importantly, the boundaries of the mentoring relationship may be less clearly defined due to the virtual nature of the relationship. To minimize these issues, as described above, we recommend establishing the mission of the meeting using an agenda. Establishing boundaries throughout the virtual mentoring relationship is also important to support success. We suggest mentors and mentees set limits on when an individual can be reached in virtual space so mentors and mentees can maintain adequate separation to enable their development.

One of the most important things that mentors can do to help mentees is to provide them with access to a wide network of professionals who will help build the career of their dreams. Whether it is potential collaborators, advisors or future colleagues, mentors can virtually connect their trainees with a strong and supportive network, which will provide tremendous and often unanticipated value at all stages in their career. Opening one's network to trainees requires a level of trust in the trainee's commitment to a given project. We encourage mentors to put trainees in contact with potential collaborators or mentors through e-mail introduction. By introducing our trainees to a network of supportive scientists, we will allow them to expand their scientific reach.

\section{Building a vision for the future}

As mentors, our goal is to prepare trainees for a successful career in science, which requires bold thinking, bravery in the face of doubt and commitment to pursuing our own hypotheses. One of the best ways to foster these traits is to encourage trainees to take risks. Mentors can help guide the next steps and directions for their students and trainees by encouraging them to ask: Are there new scientific questions we can tackle? Can trainees revisit past datasets with a new mindset to address unanswered questions? Can we mine old data to uncover new discoveries? What are the next directions to follow up on a discovery? Supporting mentees' high-risk thinking teaches them to reach higher, dream bigger and develop a deep love of science.

Silicon Valley fully understands the power of risk taking, generating trillions of dollars and transforming nearly every business sector by rewarding risk. When nurtured in a safe and supportive environment, this skill can build the character necessary to consistently achieve exponential progress. To foster this mentality, reward out-of-the-box thinking with positive reinforcement for proposing outrageous ideas or experiments. Encourage trainees to relentlessly question the dogma of every project they pursue so that they do not become trapped by walls of the past. To minimize potential risks, balance high-risk experiments with low risk projects or questions. Many principal investigators provide trainees with two projects: one 'safe' and another 'risky'. Safe projects are those that do not require difficult experiments to finish, have been de-risked with preliminary data and have sufficient expertise within the lab. This is an ideal time where both mentors and trainees can benefit working through building these aims.

Graduate students at the beginning of their journey can use this time to leap into the literature and uncover new avenues to build proposals whereas graduate students toward the end of their journey can define the final questions needed to complete their projects. Postdocs targeting the tenure track can begin defining the objectives and vision for their future research group. A majority of postdocs are sitting on data never explored, never analyzed - this is the time to data mine and find the directions that will lead to discovery at the next level. We must prepare trainees to handle the unexpected. There is no better training than providing trainees with first-hand experience in uncharted waters where they can develop the character that comes with pursuing innovative ideas. With virtual mentoring, advisors can challenge and encourage their trainees to step outside the box and envision the future of their science.

\section{Conclusions}

As scientists, we rely on the ability to plan, troubleshoot, analyze and synthesize. To keep our minds sharp and our careers moving forward, we need guidance and mentorship. Virtual mentoring is a flexible and time-efficient way to expand opportunities and refine knowledge while in quarantine. Although quarantine limits our capacity to conduct science, it can serve as a period of training during which we help each other to come out more durable and more prepared to usher in the next scientific revolution.

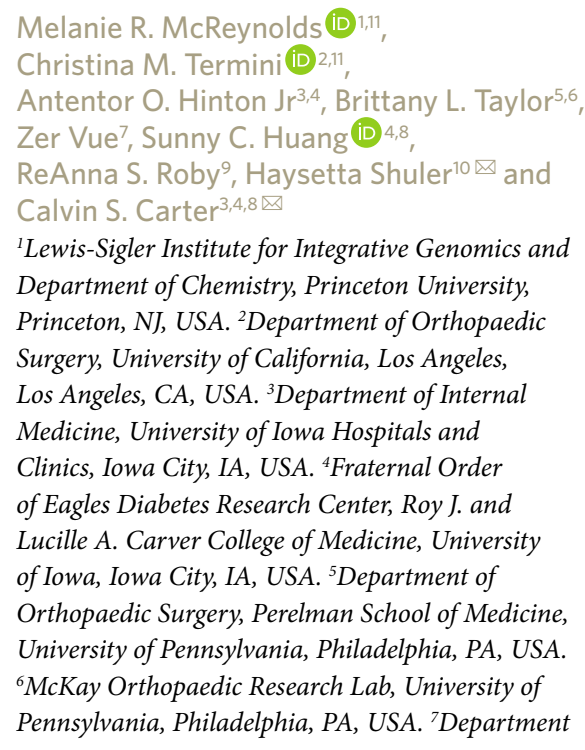


of Cell \& Tissue Biology, University of California, San Francisco, San Francisco, CA, USA. ${ }^{8}$ Geminii Inc., Iowa City, IA, USA. ${ }^{9}$ Fisk-Vanderbilt Center of Excellence, Peabody College, Vanderbilt University, Nashville, TN, USA. ${ }^{10}$ Department of Biological Sciences, Winston-Salem State University, Winston-Salem, NC, USA. ${ }^{11}$ These authors contributed equally: Melanie R. McReynolds, Christina M. Termini.

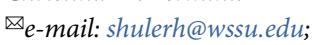

calvincarter@geminiihealth.com

Published online: 3 December 2020

https://doi.org/10.1038/s41587-020-00758-7
References

1. Kuhn, C. \& Castaño, Z. Nat. Biotechnol. 34, 781-783 (2016)

2. Leaf, G. E. Choice 45, 509 (2007).

3. Rock, D. \& Grant, H. 4, 2-5 Harv. Bus. Rev. (2016).

4. Werner-Washburne, M. Nat. Biotechnol. 36, 113-115 (2018).

5. Baugh, S. G. \& Scandura, T. A. Soc. Behav. Personal. 14, 503-521 (1999).

6. Fridkis-Hareli, M. Nat. Biotechnol. 29, 287-288 (2011).

7. Headlam-Wells, J., Gosland, J. \& Craig, J. Int. J. Inf. Manage. 26, 372-385 (2006)

Acknowledgements

The authors wish to acknowledge generous support from the following funding sources: the Burroughs Wellcome Fund Postdoctoral Enrichment Program
(M.R.M., C.M.T., A.J.H. and B.L.T.), Howard Hughes Medical Institute Hanna H. Gray Fellows Program (M.R.M.), Damon Runyon Cancer Research Foundation DRG-2327 (C.M.T.), Ford Foundation Postdoctoral Fellowship and E.E. Just Postgraduate Life Sciences Fellowship (A.J.H.), University of Pennsylvania Provost's Postdoctoral Fellowship (B.L.T.), UCSF-IRACDA Postdoctoral Fellowship K12 grant sponsored by the NIH/NIGMS (Z.V.), the Medical Scientist Training Program NIH 5T32GM007337 (S.C.H.), the National Science Foundation Award 1826755 (R.S.R.) and the American Diabetes Association Postdoctoral Fellowship 119PMF030 (C.S.C.)

Competing interests

The authors declare no competing interests. 